\title{
Diversidad cultural en las respuestas populares al Rorschach en una muestra de niños asháninkas y niños del Cusco rural
}

Lupe Jara

Pontificia Universidad Católica del Perú

\author{
Recibido: 12 de junio del 2017 / Aceptado: 19 de julio del 2017 \\ doi: https://doi.org/10.26439/persona2017.n20.1743
}

La respuesta popular es uno de los códigos del sistema comprehensivo del Rorschach más sensibles al impacto cultural; por eso, este estudio busca establecer las respuestas populares brindadas al psicodiagnóstico de Rorschach. Participaron en este estudio 108 niños, entre los 6 y 11 años, 58 asháninkas y 50 de dos comunidades rurales de Cusco. Para esta medición se ha revisado los contenidos de las 2465 respuestas generadas por los participantes. Los resultados muestran que existen tres respuestas populares: el animal entero en el DI de la lámina VIII (54,63\%), y la mariposa (44,44\%) y el murciélago (37,04\%) en la $W$ de la lámina $V$. A la vez, se encuentran diferencias en el número y contenido de la respuesta popular, de acuerdo con la comunidad, el género, la edad y el evaluador.

Rorschach / respuestas populares / niños asháninkas / niños del Cusco rural

\section{Cultural Diversity in Popular Responses to the Rorschach test in Asháninka Children and Rural Cusco Children}

The popular response is one of the Rorschach Comprehensive System codes most sensitive to cultural impact. This study seeks to establish the popular responses provided by 108 children, ages from 6 to 11, 58 asháninkas and 50 children from two rural areas in Cusco. The results show that there are three popular responses: the whole animal (D1, plate VIII, $54.63 \%)$, the butterfly (W, plate V, 44.44\%) and the bat (W, plate V, 37.04\%). At the same time, there are differences in the number and content of the popular response according to community, gender, age and the evaluator.

Rorschach / popular response / asháninkas children / rural children from Cusco

Correo electrónico: lupe.jara@pucp.edu.pe 
A lo largo de su desarrollo, el niño percibe el mundo que tiene en torno suyo con una creciente capacidad para captar su sentido, porque, conforme sus capacidades abstractas se incrementan progresivamente, su sistema perceptivo se va haciendo cada vez más especializado (Bower, 1984). En este desarrollo perceptual, el contexto cultural, social y físico influye en la focalización de la atención y en la consolidación de la capacidad discriminativa (Córdoba, 2006). Ahora bien, el Rorschach es uno de los instrumentos más completos para evaluar la organización y el funcionamiento psicológico de la persona; específicamente, permite medir el ajuste perceptivo $\mathrm{y}$ el grado de adaptación a las normas y convenciones del grupo de pertenencia del evaluado (Exner, 1994, 2000). Así, mediante las respuestas populares (P) se puede valorar el impacto de la cultura sin dejar de lado aspectos idiosincrásicos de la persona. Sin embargo, pese a que en la actualidad el sistema comprehensivo del Rorschach (SCR) cuenta con criterios estandarizados para su administración, codificación e interpretación (Exner, 1994), en el caso de los niños, muchos de estos valores son inestables (Meyer, Erdberg y Shaffer, 2007), lo que puede estar reflejando el impacto de la cultura en su proceso evolutivo. En consecuencia, resulta necesario identificar las respuestas populares en niños de diferentes contextos culturales, como el asháninka y el andino.

Conforme crece, y de acuerdo con las experiencias y expectativas que lo incentiven a actuar, el niño va definiendo cómo atiende y procesa el mundo que lo rodea (Córdoba, 2006). Para Córdoba (2006), estas experiencias y expectativas responden al tipo de problemas que se le presenten, al tipo de enseñanzas que recibe y a las habilidades que requiere dominar en su cultura. Al respecto, Timyan (1988) plantea que cada cultura pone en marcha sus propios patrones, creencias y actitudes, así como sus metas e ideales para asegurar el desarrollo intelectual, emotivo, social y moral de los niños. En tal sentido, las conductas apropiadas y esperadas en las diferentes etapas del desarrollo son conceptualizadas de un modo específico en cada cultura (Timyan, 1988). Asimismo, Córdoba (2006) considera que el contexto contribuye a focalizar la atención en lo que resulta familiar y a la pérdida de sensibilidad hacia lo que no lo es. Un ejemplo de ello lo presenta Mishra (1996, como se citó en Lafuente y Gil, 2006) al reportar una mayor diferenciación perceptual en niños de la tribu Birjia, que pasan gran parte del día en el bosque, frente a quienes se quedan en los poblados, al tener una mayor oportunidad de exploración.

Al interactuar con el entorno, el ser humano es capaz de representar a través de símbolos aspectos de la realidad, como hechos, objetos y otros símbolos y procesos, con lo que genera un cuerpo de conocimientos (Delclaux, 1982). Newell y Simon (como se citó en Delclaux, 1982) agregan que al procesar la información se comparan ocurrencias de símbolos y de estructuras simbólicas; y se clasifica, almacena y recupera tales símbolos $\mathrm{y}$ estructuras simbólicas, al mismo tiempo que se crean otras nuevas a partir de modificar 
las existentes. Este procesamiento permite establecer el vínculo entre la percepción sensorial y nuestros conocimientos para crear así representaciones mentales (Delclaux, 1982).

Exner (2000), por su parte, plantea que este procesamiento involucra las estrategias y operaciones que inducen al ingreso de la información en el sistema mental, lo que implica la exploración de un campo estimular, y la creación, en la memoria a corto plazo, de unas imágenes (íconos) de ese campo o de partes del mismo. A la vez, este autor considera que durante la recepción de los datos nuevos e inestructurados se manifiestan de manera particular las estrategias generales que la mayoría de las personas desarrolla.

Justamente, un instrumento que provee estímulos nuevos e inestructurados es el psicodiagnóstico de Rorschach, prueba creada por Hermann Rorschach en 1921. Esta prueba, conformada por diez láminas con manchas de tinta sobre fondo blanco, es considerada una de las más útiles y valiosas para evaluar el funcionamiento psicológico de las personas; por ese motivo, es una de las más usadas e investigadas en el mundo (Exner, 1994), situación a la que nuestro país no es ajeno (Ráez, 2006). Pese a ello, no es hasta que Exner y numerosos investigadores inician un largo proceso de ensamblaje e investigación para crear el SCR que se determinan sus propiedades psicométricas, proveyéndole de los estándares de validez y confiabilidad requeridos por una prueba psicológica (Exner, 1994).

De esta manera, el SCR estandariza los criterios y pautas para la administración, codificación e interpretación de la prueba (Exner, 1994). Pero ¿cómo un grupo de respuestas verbales ante unas manchas puede revelar aspectos importantes de la personalidad? (Smith, 1994). Exner (2000) plantea que la naturaleza de la tarea implicada en el Rorschach estimula un conjunto complejo de operaciones y mecanismos psicológicos que se activan en el proceso de toma de decisiones ante las manchas, tal como sucede en la toma de decisiones rutinarias de la vida cotidiana. En tal sentido, las respuestas a las manchas informan sobre los aspectos cognitivos, afectivos y relacionales del evaluado. Como señala Piotrowski (como se citó en Sendín, 2007), "la genialidad de Rorschach consistió en descubrir que se podían deducir los rasgos esenciales de una persona a través del estudio de sus peculiaridades perceptivas" (p. 288). De ahí que, en la actualidad y a nivel mundial, el SCR sea considerado uno de los instrumentos más completos para evaluar la organización y el funcionamiento psicológico de las personas (Exner, 2000). Y es justamente su consolidación y expansión internacional lo que lleva a cuestionar si el SCR, creado y normado con base en una muestra de sujetos norteamericanos, ¿puede ser usado con personas de una cultura diferente?

$\mathrm{Al}$ respecto, uno de los códigos del SCR que parece más influenciado por la variable cultural es la respuesta popular. Esta se refiere a los contenidos que se encuentran en al menos uno de cada tres protocolos (Exner, 1994). Actualmente, el SCR comprende un listado de 13 respuestas populares. La tarea de identificar un 
contenido apropiado a las características perceptivas de la mancha, siendo las respuestas populares las más fáciles de hallar al responder a los rasgos estimulares más distintivos de las manchas, informa sobre el grado de adecuación perceptiva del sujeto y de la adaptación a las normas y convenciones de su grupo de pertenencia (Exner, 2000).

En este procesamiento, es importante considerar que las láminas del Rorschach no fueron diseñadas por su autor de manera fortuita, por lo que lejos de ser completamente ambiguas presentan ciertas características que limitan el conjunto de respuestas posibles, a la vez que fomentan tipos específicos de respuestas (Exner, 2000). Exner (1994, 1996, 2000) plantea que en la tarea de desidentificar los estímulos para procesar las respuestas se realiza una representación interna del campo estimular para clasificarlo — global o parcialmente - como suficientemente similar a objetos conocidos o imaginados; y se generan así respuestas potenciales. Entre estas características, Exner (2000) llama a las más potentes indicios distales críticos (el más evidente es la forma). Al respecto, al usar los indicios más distintivos de las manchas, para numerosos sujetos se facilita su decodificación y coinciden en las respuestas en las mismas áreas; el resultado son las respuestas populares (Sendín, 2007).

El hecho de que una de cada tres personas frente a un conjunto de estímulos nuevos e inestructurados, como los que conforman el psicodiagnóstico de Rorschach, evoque en ciertas zonas en cada lámina las mismas respuestas habla de la probabilidad de ver el mundo como lo hacen los demás (Sendín, 2007). Coherentemente, la frecuencia de las respuestas populares aumenta en forma gradual a medida que el niño crece (Ames, Learned, Metraux y Walter, como se citó en Exner, 1994), aunque el sistema comprehensivo no especifica cuáles son las populares que aparecen primero y cuáles son las que se van agregando. A la vez, si bien la respuesta popular es uno de los signos más estables en el protocolo, algunos estudios transculturales reportan diferencias con respecto al promedio y a la aparición de respuestas populares específicas (Exner, 1994).

Como consecuencia de lo anterior, la frecuencia de las respuestas populares es bastante elevada (Exner, 1994), y si bien el sistema comprehensivo mantiene el criterio establecido por Rorschach de definirlas a partir de su presencia en uno de cada tres protocolos (Shaffer, Erdberg y Meyer, 2007), Exner (2000) considera que este criterio es "muy conservador" (p. 235), pues la mayoría de las $P$ tienen una presencia bastante mayor. De hecho, este autor encuentra que solo dos populares se encuentran por debajo del $40 \%$ : la figura humana o antropomórfica en el área D3 de la lámina IX (35 \%) y la cabeza de animales o animales enteros en el área D1 de la lámina II (39 \%). En tanto, las P con una mayor frecuencia son las siguientes: el animal cuadrúpedo en el área D1 de la lámina VIII (por encima del $90 \%$ ), la figura humana en el D9 de la lámina III (mayor al $80 \%$ ) y las respuestas de mariposa o murciélago a la $\mathrm{W}$ de la lámina $\mathrm{V}$, ambas con una proporción similar (44 \% y $41 \%$, respectivamente). 
Al respecto, si bien todos los sistematizadores del Rorschach han considerado importante identificar las respuestas populares, los criterios para establecer las mismas han sido diferentes (Exner, 1994). Por ejemplo, Alessandro, Alonso y Passalacqua (2000), para el sistema argentino, consideran $\mathrm{P}$ a las respuestas que aparecen en el $25 \%$ o más de la población y como $(\mathrm{P})$ a las dadas entre el $15 \%$ y el $24 \%$. Frente a las críticas que señalan que el criterio de un tercio puede ser restrictivo, Exner (1994) responde que, además de este criterio, se encuentra el estadístico, pues las P incluidas en el SCR se diferencian de otras respuestas con una frecuencia alta. Así, aunque la $\mathrm{P}$ de la lámina IX es la de menor presencia, su frecuencia es mayor que la siguiente respuesta más usual (la figura humana del área D4 de la lámina I, que tiene una frecuencia del $25 \%$ ), la cual no se diferencia de otras respuestas frecuentes (como la cara animal o máscara en WS de la lámina I [24\%], cohete en el DS5 de la lámina II [19\%], mariposa en el D3 de la lámina III, piel de animal en la W de la lámina IV [22\%] y poste totémico en el D3 de la lámina VI [18\%]; existen también otras 50 respuestas que aparecen entre el $13 \%$ y el $17 \%$ ).

Sin embargo, aun manteniendo el criterio de un tercio, la presencia de las respuestas populares ha variado en el mismo SCR; así, de la primera lista publicada en 1974, luego de una década se han eliminado cinco y se han incorporado dos nuevas (Exner, 1994), de manera que el listado actual consta de 13 respuestas populares (Exner, 2001). De acuerdo con
Exner (1994), citando a varios autores (Bourguinon y Nett, 1955; Hallowell, 1956), esta lista básica parece mantenerse en otros grupos culturales. De ahí que, aunque otros investigadores citados por Exner (Leighton y Kluckholm, 1948; Honigmann, 1948; Joseph y Murria, 1951; Fried, 1977) hayan encontrado populares específicas en determinadas culturas, estas en general se añaden — sin sustituir - a las contenidas en la lista básica. Para Exner (1994), lo anterior queda corroborado por un estudio conducido por la Fundación para la Investigación del Rorschach con sujetos de 12 países, el cual encuentra que 11 de las 13 respuestas populares aparecen al menos en el $40 \%$ de los protocolos, quedando por debajo del criterio las respuestas populares de las láminas IX (26 \%) y II (31 \%); por esa razón, este autor considera que es improbable que las respuestas con mayor frecuencia sufran variaciones transculturales.

Pese a lo anterior, otros estudios reportan no solo la aparición de $\mathrm{P}$ específicas, sino también la sustitución o eliminación de algunas populares, en particular - como es previsible- de las $\mathrm{P}$ menos frecuentes. Así, no existe respuesta popular en la lámina IX para muestras de diferentes países, como la española (Sendín, como se citó en Exner, 2001), la japonesa (Nakamura, Fuchigami y Tsugawa, 2007; Tsugawa et al., como se citó en Lunazzi, 2006) y la argentina (Lunazzi, 2006), entre otras. En el caso de la lámina II, se elimina la P del SCR en una muestra venezolana (Ephraim, como se citó en Lunazzi, 2006); se la 
sustituye por "dos personas" en una muestra japonesa (Nakamura et al., 2007) y por "elfos de navidad" en sujetos finlandeses (Fried, como se citó en Exner, 1994: Mattlar, como se citó en Lunazzi, 2006), y se cambia alguno de los animales específicos, como "cordero" por "conejo" en una muestra española (Sendín, como se citó en Exner, 2001) y por "toro" en sujetos argentinos (Lunazzi, 2006).

A lo anterior se agregan otras variaciones culturales en el resto de las respuestas populares del SCR. Así, en la lámina $\mathrm{I}$, se elimina la $\mathrm{P}$ mariposa en sujetos japoneses (Nakamura et al., 2007; Tsugawa et al., como se citó en Lunazzi, 2006), venezolanos (Ephraim, como se citó en Lunazzi, 2006) y finlandeses (Mattlar, como se citó en Lunazzi, 2006). En la lámina III, solo aparece como $\mathrm{P}$ la figura humana (no su representación) en sujetos japoneses (Nakamura et al., 2007; Tsugawa et al., como se citó en Lunazzi, 2006) y finlandeses (Mattlar, como se citó en Lunazzi, 2006). En la lámina $\mathrm{IV}$, solo es $\mathrm{P}$ la figura parahumana (no la humana) en una muestra argentina (Lunazzi, 2006), española (Sendín, como se citó en Exner, 2001) y japonesa (Tsugawa et al., como se citó en Lunazzi, 2006); se halló que también aparecía como P la piel de animal en la muestra española (Sendín, como se citó en Exner, 2001) y finlandesa (Mattlar, como se citó en Lunazzi, 2006). En la lámina VI, en dos muestras japonesas (Nakamura et al., 2007; Tsugawa et al., como se citó en Lunazzi, 2006) aparece una segunda P: instrumento musical. En la lámina VII, no hay $\mathrm{P}$ en una muestra finlandesa
(Mattlar, como se citó en Lunazzi, 2006). $\mathrm{Y}$, en la lámina $\mathrm{X}$, no existe popular para sujetos japoneses (Nakamura et al., 2007; Tsugawa et al., como se citó en Lunazzi, 2006) y finlandeses (Mattlar, como se citó en Lunazzi, 2006).

En esa misma línea, en una muestra de 100 niños del departamento de Apurímac, Jara (2006) encuentra que del listado de 13 respuestas populares del SCR, los niños andinos solo brindan cuatro de ellas: la figura humana de la lámina III, la mariposa de la lámina $\mathrm{V}$, el animal de la lámina VIII y la araña de la lámina $X$, pero se eleva la frecuencia de contenidos propios de su contexto, tales como cóndor, oveja, humo, entre otros. Esta misma autora (Jara, 2015), en una muestra de 346 niños limeños, halla cinco respuestas P: la figura humana en el D9 de la lámina III (40,7 \%), el lazo, moño o corbata en el D3 de la lámina III (39,3\%), la mariposa (51,5\%) y el murciélago $(47,1 \%)$ en la $\mathrm{W}$ de la lámina $\mathrm{V}$; y el animal entero en el D1 de la lámina VIII (55,5\%).

Como se aprecia, la respuesta popular parece muy influida por la cultura, y constituye un indicador sobre el contacto con la realidad (Alessandro et al., 2000) y el grado de consenso que el sujeto alcanza con su medio en la representación de esa realidad. De ahí que el SCR encuentra que el número de respuestas populares dentro del promedio (entre 5 y 8, con una media cercana a 7) indica que, ante situaciones obvias, se generarán respuestas concordantes con las expectativas sociales (Exner, 1994). El incremento de las $\mathrm{P}$ puede revelar una particular preocupación por detectar las claves de las conductas socialmente 
aceptables y convencionales, como también una tendencia al perfeccionismo (Exner, 2000), lo que a menudo implica un sacrificio de las capacidades creativas y los rasgos más personales y únicos del individuo (Exner y Sendín, 1998; Sendín, 2007). En cambio, cuando $P$ disminuye, es probable que se responda de una manera menos convencional, incluso ante situaciones simples y bien definidas (Exner, 2000); aunque también puede revelar a una persona singular y original que, sin violar la realidad, prefiere manejarla de forma más individualista (Exner y Sendín, 1998; Sendín, 2007).

En cuanto al género, Exner (1994) señala que existen diferencias en algunas populares. Así, en las láminas I y V, las mujeres dan la $\mathrm{P}$ de mariposa más que los varones, quienes por su parte mencionan más la de murciélago que ellas. En la lámina VII, las mujeres reportan más veces mujeres, mientras que los hombres ven más niños o indios. En la lámina VIII, los varones perciben con mayor frecuencia animales grandes y salvajes, en tanto que las mujeres brindan más respuestas de animales domésticos y pequeños. $\mathrm{Y}$, finalmente, en la lámina $\mathrm{X}$, los hombres proporcionan más la respuesta de cangrejo, y ellas dan más la respuesta de araña.

De otro lado, considerando el desarrollo evolutivo, Ames, Learned, Metraux y Walter (como se citó en Exner, 1994) encuentran que la frecuencia de las $\mathrm{P}$ aumenta gradualmente a medida que el niño crece, lo que es confirmado por el SCR. Así, la media de P pasa de 4,66 a los cinco años a 6,06 a los 11 años (Exner, 2001). Sin embargo, el SCR no indica cuáles son las $\mathrm{P}$ esperadas en los niños, ni cuáles se van agregando conforme crece.

Teniendo en cuenta el impacto de la cultura en la definición de las respuestas populares, diferentes autores (Alessandro et al., 2000; Lunazzi, 2006) plantean la necesidad de identificar estas respuestas en el contexto específico de la evaluación. Ello resulta fundamental, sobre todo en el caso de los niños, para quienes la cultura de origen -en contraste con la cultura hegemónica - tiene un mayor peso en su concepción del mundo. En el caso de los niños, Exner y Weiner (1995) consideran, además, que la comprensión de la data debe enmarcarse dentro de lo esperado evolutivamente. Lo anterior representa una tarea compleja, ya que con los niños la interpretación se transforma en un gran reto, debido a que los rápidos cambios que ocurren mientras ellos crecen generan una amplia gama de diferencias individuales en casi cada año del ciclo evolutivo.

De otro lado, si bien Exner y Weiner (1995) piensan que las pautas para la administración del Rorschach, los criterios para su posterior codificación y los principios para su interpretación son transversales a todos los grupos de edad, estudios recientes indican la necesidad de una adaptación del SCR en el caso de los niños. Así, Jara (2011) comenta que, en su experiencia de aplicación del Rorschach a niños cuya lengua materna es el quechua, ha notado que la calidad de la interacción cambia marcadamente al ser presentada por alguien que cumple un rol valorado en la comunidad, al saludarlos y preguntarles sus nombres en quechua, y al mostrar algún conocimiento sobre el 
lugar donde viven y las actividades que realizan; así, la interacción se vuelve más cercana y confiable. Esto habla de la necesidad de dedicar un especial interés al establecimiento del rapport con los niños antes de administrar la prueba.

Por otra parte, respecto a los criterios para la codificación de las respuestas, actualmente Meyer et al. (2007) han logrado consolidar una data normativa internacional para el SCR, que incluye muestras de adultos de 16 países (Argentina, Australia, Bélgica, Brasil, Dinamarca, España, Estados Unidos, Finlandia, Grecia, Israel, Italia, Japón, Países Bajos, Perú, Portugal y Rumania). Sin embargo, estos autores observan que, en el caso de los niños y adolescentes, en diferentes muestras y países, los valores en varios códigos del SCR son inestables $\mathrm{y}$ frecuentemente muy distintos.

Lo anterior parece confirmar la advertencia planteada por Allen y Dana (2004) acerca de incorporar las variables culturales antes de asumir la universalidad de instrumentos construidos en otras sociedades. A la vez, dado que la data, como afirma Weiner (2004, como se citó en Dana, 2009), es el vehículo primario para la interpretación, es necesario que se adapte al contexto donde se utiliza. En efecto, tanto Allen y Dana (2004) como Daroglou (2009) enfatizan la importancia de adoptar una perspectiva cultural, ya que existe el riesgo de considerar que la interpretación estándar del SCR puede ser aplicada en todo el mundo.

Por su parte, Franchi y AndronikofSanglade (1999) llaman la atención sobre las dificultades metodológicas que genera esta aproximación, lo que se refleja en la ausencia de significados normativos para las diferentes culturas y grupos subculturales, así como en las dificultades inherentes al desarrollo de la data normativa. Sin embargo, Ephraim (1996) considera que los aportes metodológicos del SCR facilitan la realización de estudios transculturales, pues se basan en un método de investigación sólido.

Como vemos, si bien el Rorschach aplicado a niños provee una imagen de cómo van construyendo sus representaciones mentales en torno al contexto donde se desarrollan, ajustándose a sus normas y convenciones conforme van creciendo, resulta necesario identificar cuáles son las respuestas populares específicas que ellos brindan, a fin de interpretar correctamente los resultados que arroja esta prueba. Esto es particularmente relevante si se constata que el Rorschach es una de las pruebas más empleadas por los psicólogos para la evaluación de la personalidad (Ráez, 2006), además de ser el instrumento principal en varias investigaciones realizadas con niños (Alcalde, 2001; Ampuero, 2000; Del Castillo, 2014; Cornejo, 2005; Eldad, 2009; Jara, 2000; Puga, 2008; Rivarola, 2015; Rivera, 2000; Vargas, 2003; entre otros). A partir de lo expuesto, este estudio busca establecer las respuestas populares en los niños asháninkas $\mathrm{y}$ andinos, y de manera específica explorar si existen diferencias en la $\mathrm{P}$ de acuerdo con el sexo, la edad y la comunidad. Para ello, se administró el psicodiagnóstico de Rorschach a menores entre los 6 y 11 años de edad de estas comunidades. 
Tabla 1

Distribución de los participantes

\begin{tabular}{lccccc}
\hline & \multicolumn{2}{c}{ Asháninkas } & \multicolumn{2}{c}{ Cusqueños } & \\
\cline { 2 - 5 } Edad & Niños & Niñas & Niños & Niñas & Total \\
\hline 6-7 años & 8 & 8 & 5 & 4 & 25 \\
8-9 años & 10 & 10 & 13 & 9 & 42 \\
10-11 años & 12 & 10 & 8 & 11 & 41 \\
Total & 30 & 28 & 26 & 24 & 108 \\
\hline
\end{tabular}

\section{Método}

\section{Participantes}

Participaron en este estudio 108 niños entre los 6 y 11 años, 58 asháninkas y 50 de dos comunidades rurales de Cusco. Los niños asháninkas pertenecen a las comunidades en la provincia de Chanchamayo (Alto Perené), en el departamento de Junín. Por su parte, los niños de Cusco pertenecen a dos comunidades rurales, una de ellas ubicada en el distrito de Lares, en la provincia de Calca; y la otra en el distrito de Tinta, en la provincia de Canchis. El contacto con sus familias se realizó a través de personas o instituciones con las cuales tienen una relación de confianza; y se solicitó el consentimiento informado de los padres para la participación de sus hijos e hijas. En el caso de los niños asháninkas, se solicitó también el permiso de las autoridades comunales.

Dadas las dificultades de acceso a los participantes, se trata de una muestra no probabilística de tipo incidental, ya que la conformación de la misma dependió de la disponibilidad de los participantes (Hernández, Fernández y Baptista, 2010).

\section{Medición}

La medición de las respuestas populares se realizó utilizando el psicodiagnóstico de Rorschach. Se tomó como criterio que la respuesta aparezca en uno de cada tres protocolos, según lo propuesto por Rorschach y retomado por diferentes sistematizadores, entre ellos Exner (1994), para el SCR. A la vez, siguiendo a Exner, se incluyó también como criterio que estas respuestas se diferencien de otras respuestas con una frecuencia alta.

La codificación de las respuestas populares es relativamente fácil, y es uno de los códigos menos expuestos a las influencias adulterantes por parte del examinador (Baughman, como se citó en Exner, 1994). De ahí que Exner reporte que la fiabilidad entre codificadores para la respuesta popular muestra un nivel de concordancia de alrededor del 99 \%. León (1983), por su parte, reseña diferentes estudios que avalan una alta confiabilidad intercalificador y test-retest de la P. No hay, por tanto, lugar para el error; las discrepancias, cuando existen, se deben más a las omisiones que a los desacuerdos. 


\section{Procedimiento}

Para este estudio, se buscó la participación de niños y niñas de dos contextos culturales distintos: el asháninka (Junín) y el andino (Cusco). En el caso de los menores asháninkas, se contactó a los representantes y las autoridades comunales; una de estas comunidades solicitó, además, la firma de un convenio institucional entre ella y la Pontificia Universidad Católica del Perú, a fin de documentar la realización de la investigación. A las autoridades comunales se les planteó el proyecto de investigación, solicitándoles su autorización para el ingreso de la investigadora a la comunidad, así como para invitar a las familias a ser parte del estudio. En total, se realizaron tres viajes a esta comunidad; en el último de ellos, la investigadora y una asistente ${ }^{1}$ fueron alojadas en una de las casas de la comunidad. Dado que el número de niños era bajo, posteriormente se realizaron nuevas coordinaciones con otras comunidades de la zona para ampliar el número de participantes; en ellas nuevamente el contacto inicial se hizo con las autoridades comunales.

En cambio, en el caso de los niños andinos, el contacto se produjo a través de representantes de la Iglesia católica que llevan a cabo un trabajo pastoral de larga data en la zona y que, por lo tanto,

1 La asistente es una psicóloga joven que ha trabajado antes con la investigadora en un estudio sobre el Rorschach en niños limeños. Ha obtenido su licenciatura con una tesis que utiliza el Rorschach en niños. Además, capacita a los estudiantes en el manejo de la prueba. cuentan con la confianza de la población. Los agentes pastorales convocaron a los padres y madres de familia con hijos e hijas entre los 6 y 11 años de edad para explicarles el propósito del estudio y solicitarles su consentimiento para la participación de los menores. En Cusco, la administración del Rorschach lo realizó únicamente la investigadora.

A los padres y madres de familia se les informó quién era la investigadora y que la participación de sus hijos e hijas era estrictamente voluntaria, por lo que si deseaban interrumpir su evaluación, podían hacerlo en cualquier momento, sin que ello los perjudicase en ninguna forma. Del mismo modo, se les comunicó que la información recogida sería manejada de manera confidencial y que no se usaría para ningún otro fin fuera de la investigación. Y, con relación a la confidencialidad, la investigadora se comprometió a que la información brindada por los menores a las pruebas sería codificada usando un número de identificación $\mathrm{y}$, por lo tanto, serían anónimas. Se buscó usar un lenguaje sencillo, no técnico, para explicar estos aspectos.

Los elementos sobre los cuales los padres y las madres de familia otorgaron su consentimiento informado fueron estos: que se llevaría a cabo una reunión de aproximadamente una hora, en la que en un primer momento se buscaría establecer una relación de confianza con el o la menor, a fin de que se sienta cómodo(a). Se les explicó, además, que no existían respuestas buenas o malas. También se les indicó que si tenían 
alguna pregunta sobre la participación de sus hijos en el estudio, podían comunicarse con la investigadora a través de la persona o institución que facilitó el contacto entre ellos y la misma. Solo en un caso, una madre de familia consultó a la investigadora si la participación de su hijo en este estudio sería remunerada, al responderle que no, igualmente consintió en la participación del menor.

En el caso de los niños asháninkas, se ofreció a las autoridades comunales que, al término del estudio, se realizaría una charla en la que se devolverían los resultados encontrados en el grupo de niños, a la par que se les propuso organizar una charla sobre algún aspecto de la crianza de sus hijos que ellos quisieran conocer o profundizar. Mientras que, en el caso de los niños andinos, se prometió a las autoridades religiosas contactadas la devolución de los resultados al término del estudio.

Para la administración de la prueba se estandarizó un procedimiento que enfatiza al comienzo de la evaluación el trabajo de rapport con los niños, para lo cual se hicieron dibujos y una breve entrevista. Con ello, se espera que el niño se familiarice con la evaluadora y con la situación de evaluación. En un segundo momento, se efectuó la administración del Rorschach.

En total, se administró la prueba a 119 menores, 56 provenientes de dos comunidades andinas cusqueñas y 63 provenientes de diferentes comunidades asháninkas. De estos protocolos, se eliminaron 8 que pertenecían a niños fuera del rango de edad requerido: 3 niños asháninkas de 5 años y 5 niños de 12 años (uno asháninka y cuatro andinos); y 3 cuyo número de respuestas fue menor a 14 (una niña asháninka de 6 años, una niña andina de 6 años y un niño andino de 9 años). Así quedaron para este estudio 108 protocolos.

Una vez aplicados los protocolos, la autora codificó todos los contenidos brindados por los menores, para establecer cuáles de ellos cumplían con el criterio de estar presentes en uno de cada tres protocolos, a fin de ser considerada una respuesta popular. Posteriormente, los datos fueron analizados mediante estadísticos descriptivos y comparativos. Adicionalmente, se aplicó la prueba Z para medir si la proporción observada cumplía de manera significativa con el criterio establecido para las respuestas populares, más allá de la muestra de participantes. Del mismo modo, se empleó la prueba chi-cuadrado $\left(X^{2}\right)$ para explorar diferencias por sexo, edad, comunidad y evaluador.

\section{Resultados}

A continuación se presentan los resultados, que se basan en las 2465 respuestas codificadas en los 108 participantes, con un promedio de 22,82 respuestas por protocolo.

En la tabla2, se puede observar que tres respuestas cumplen con el criterio de estar presentes en uno de cada tres protocolos. Estas respuestas son las siguientes: en la lámina VIII, el animal entero en el D1 $(54,63 \%) \mathrm{y}$, en la lámina $\mathrm{V}$, la mariposa $(44,44 \%)$ y el murciélago $(37,04 \%)$ en la 
W. Sin embargo, aplicando la prueba Z, para medir si la proporción de la muestra cumple de manera significativa con el criterio establecido para las respuestas populares más allá de ella, se encontró que solo las dos primeras lo cumplen.

Otra respuesta que alcanzaría el criterio para ser considerada popular es la figura parahumana $(33,33 \%)$ en la lámina IV, si se agrupan las respuestas dadas a la $\mathrm{W}$ y al D7, tal como sucede en el SCR. Sin embargo, la frecuencia de la figura parahumana es mucho mayor en $\mathrm{W}(24,07 \%)$ que en el D7 $(9,26 \%)$. A la vez, no se ha incluido en esta agrupación a la respuesta de la figura humana, por considerarse que su presencia es baja y que difiere conceptualmente de la figura parahumana. Del mismo modo, aunque las respuestas de murciélago (25\%) y mariposa $(16,67 \%)$ en la lámina $\mathrm{I}, \mathrm{y}$ la araña $(27,8 \%)$ y el cangrejo $(10,2 \%)$ en la lámina $\mathrm{X}$, al agruparse, cumplirían con el criterio de un tercio, por tratarse de contenidos conceptuales distintos y por obtener una frecuencia mayor al 33,3\% de manera individual en el SCR, se ha optado por mantener este tratamiento singularizado de las mismas.

En el caso del grupo de niños asháninkas, se han codificado 1215 respuestas en 58 participantes, con un promedio de
20,95 respuestas por protocolo. En la tabla 4 , se puede observar que cuatro respuestas cumplen con el criterio de estar presentes en uno de cada tres protocolos: el animal entero en el D1 $(58,62 \%)$ de la lámina VIII, el murciélago (50 \%) y la mariposa $(44,83 \%)$ en la $\mathrm{W}$ de la lámina $\mathrm{V}$, y el murciélago en la W de la lámina I (37,9\%). De ellas, solo las tres primeras presentan una proporción que resulta significativa más allá de la muestra.

En el caso del grupo de niños andinos, se han codificado 1250 respuestas en 50 participantes, con un promedio de 25 respuestas por protocolo. En la tabla 5, se puede observar que solo dos respuestas cumplen con el criterio de estar presentes en uno de cada tres protocolos: el animal entero en el D1 de la lámina VIII (50 \%) y la mariposa en la $\mathrm{W}$ de la lámina V (44 \%); pero solo la primera tiene una proporción que resulta significativa más allá del grupo de participantes.

De otro lado, usando la prueba chicuadrado $\left(X^{2}\right)$, se encuentra que la respuesta de murciélago de la lámina $\mathrm{V}$ presenta una diferencia significativa $(p<.05)$ en cuanto a la comunidad, pues está más presente $\mathrm{y}$ es una respuesta popular entre los niños asháninkas (50\%), en contraste con los niños andinos del Cusco (22 \%).

Tabla 2

Respuestas populares en el grupo de niños asháninkas y andinos

\begin{tabular}{lllccc}
\hline Lámina & Localización & Respuesta & $\%$ & $Z$ & Sig. \\
\hline VIII & D1 & Figura animal entera & 54,63 & 4,69 & $0,00^{*}$ \\
\hline V & W & Mariposa & 44,44 & 2,45 & $0,01^{*}$ \\
& W & Murciélago & 37,04 & 0,82 & 0,21 \\
\hline
\end{tabular}


Tabla 3

Respuestas populares en el sistema comprehensivo con una frecuencia alta en la muestra de participantes

\begin{tabular}{cclc}
\hline Lámina & Localización & & Respuesta \\
\hline I & W & Murciélago & 25 \\
& W & Mariposa & 16,67 \\
\hline III & D9 & Figura humana & 28,7 \\
\hline IV & W & Figura parahumana & 24,07 \\
& D7 & Figura parahumana & 9,26 \\
\hline V & W & Mariposa & 44,44 \\
& W & Murciélago & 37,04 \\
& W & Ave & 25 \\
\hline VIII & D1 & Figura animal entera & 54,63 \\
\hline X & D1 & Araña & 27,78 \\
& D1 & Cangrejo & 10,19 \\
\hline
\end{tabular}

Tabla 4

Respuestas populares en el grupo de niños asháninkas

\begin{tabular}{cclccc}
\hline Lámina & Localización & \multicolumn{1}{c}{ Respuesta } & $\%$ & $Z$ & Sig. \\
\hline VIII & D1 & Figura animal entera & 58,62 & 4,09 & $0,00^{*}$ \\
\hline V & W & Murciélago & 50,00 & 2,69 & $0,00^{*}$ \\
& W & Mariposa & 44,83 & 1,86 & $0,03^{*}$ \\
\hline I & W & Murciélago & 37,93 & 0,74 & 0,23 \\
\hline
\end{tabular}

${ }^{*} p<.05$

Tabla 5

Respuestas populares en el grupo de niños andinos

\begin{tabular}{cclccc}
\hline Lámina & Localización & \multicolumn{1}{c}{ Respuesta } & $\%$ & $Z$ & Sig. \\
\hline VIII & D1 & Figura animal entera & 50,00 & 2,50 & $0,01^{*}$ \\
\hline V & W & Mariposa & 44,00 & 1,60 & 0,05 \\
\hline
\end{tabular}

${ }^{*} p<.05$ 
En cuanto al sexo, se encuentra que tanto para los varones como para las mujeres tres respuestas cumplen con el criterio para ser consideradas respuestas populares: el animal entero en el D1 en la lámina VIII (60,7\% en los varones y 48,1 \% en las mujeres), la mariposa en la $\mathrm{W}$ de la lámina $\mathrm{V}(51,8 \%$ en los varones y $36,5 \%$ en las mujeres) y el murciélago en la $\mathrm{W}$ de la lámina $\mathrm{V}(39,3 \%$ en los varones y 34,6\% en las mujeres). Sin embargo, en el caso de los varones, solo las dos primeras tienen una proporción que resulta significativa más allá del grupo de participantes; en cambio, en el caso de las mujeres, solo el animal entero en el D1 en la lámina VIII tiene una presencia significativa más allá de la muestra. De otro lado, es importante destacar que, usando la prueba chicuadrado $\left(X^{2}\right)$, se encuentra que no existen diferencias significativas por sexo en ninguna de las respuestas más frecuentes dadas por los menores a la prueba.

Al considerar la edad, se aprecia que en todos los grupos de edad tres respuestas alcanzan el criterio para ser consideradas populares; de ellas, el animal entero en el D1 en la lámina VIII y la mariposa en la $\mathrm{W}$ de la lámina $\mathrm{V}$ son $P$ entre los 6 y 11 años. En tanto, entre los 6 y 7 años resulta $P$ la figura parahumana en la W de la lámina IV; mientras que entre $\operatorname{los} 8$ y 11 años esta respuesta deja de ser $\mathrm{P}$ y es reemplazada por la respuesta de murciélago. Sin embargo, al considerar qué proporciones resultan significativas más allá de la muestra, encontramos que entre los menores de 6 y 7 años ninguna respuesta logra cumplir el criterio de manera significativa; en el caso de los niños de 8 y 9 años, solo lo consigue la respuesta del animal entero en el D1 en la lámina VIII, y para los niños de 10 y 11 años, las respuestas del animal entero en el D1 en la lámina VIII y la mariposa en la $\mathrm{W}$ de la lámina $\mathrm{V}$. De otro lado, es importante indicar que, de acuerdo con la prueba chi-cuadrado $\left(X^{2}\right)$, no existen diferencias significativas por grupos de edad.

Tabla 6

Respuestas populares por sexo

\begin{tabular}{cclccc}
\hline Lámina & Localización & \multicolumn{1}{c}{ Respuesta } & Niño & Z & Sig. \\
\hline VIII & D1 & Figura animal entera & 60,7 & 4,35 & $0,00^{*}$ \\
\hline V & W & Mariposa & 51,8 & 2,93 & $0,00^{*}$ \\
& W & Murciélago & 39,3 & 0,94 & 0,17 \\
\hline Lámina & Localización & Respuesta & Niña & $Z$ & Sig. \\
\hline VIII & D1 & Figura animal entera & 48,1 & 2,26 & $0,01^{*}$ \\
\hline V & W & Mariposa & 36,5 & 0,49 & 0,31 \\
& W & Murciélago & 34,6 & 0,20 & 0,42 \\
\hline
\end{tabular}

${ }^{*} p<.05$ 
Finalmente, se consideró conveniente analizar el efecto del evaluador sobre las respuestas populares en el grupo de niños asháninkas, donde los protocolos fueron administrados por la investigadora y una asistente. Aquí se encontró que la respuesta de la araña en el D1 de la lámina $\mathrm{X}$ es significativamente mayor $(p<.05)$ cuando la evaluadora es la investigadora (45,5\% versus $11,1 \%)$. De otro lado, aunque no llegan a ser diferencias significativas, se observa que tres respuestas más alcanzan el criterio de estar presentes en uno de cada tres protocolos para el grupo de participantes, cuando la evaluadora es la investigadora: el murciélago en la $\mathrm{W}$ de la lámina $\mathrm{I}$, la figura humana en el D9 de la lámina III y la figura parahumana en la $\mathrm{W}$ de la lámina IV. Otro dato importante al respecto es el promedio de respuestas obtenido, siendo este significativamente mayor cuando los protocolos son administrados por la investigadora (media total $=24,92$, mediana $=22,5$ y $\operatorname{moda}=17$; media asháninka $=24,73$ y media andinos $=25)$, en contraste con la asistente (media $=$ $18,64$, mediana $=15$ y moda $=14)$.

Tabla 7

Respuestas populares por edad

\begin{tabular}{cclccc}
\hline Lámina & Localización & \multicolumn{1}{c}{ Respuesta } & $6-7$ años & Z & Sig. \\
\hline VIII & D1 & Figura animal entera & $48,0 \%$ & 1,56 & 0,06 \\
\hline V & W & Mariposa & $40,0 \%$ & 0,71 & 0,24 \\
\hline IV & W & Figura parahumana & $36,0 \%$ & 0,28 & 0,39 \\
\hline Lámina & Localización & Respuesta & $8-9$ años & Z & Sig \\
\hline VIII & D1 & Figura animal entera & $57,1 \%$ & 3,273 & $0,00^{*}$ \\
\hline V & W & Mariposa & $40,5 \%$ & 0,98 & 0,16 \\
& W & Murciélago & $38,1 \%$ & 0,65 & 0,26 \\
\hline Lámina & Localización & Respuesta & $10-11$ años & Z & Sig \\
\hline VIII & D1 & Figura animal entera & $56,1 \%$ & 3,09 & $0,00^{*}$ \\
\hline V & W & Mariposa & $51,2 \%$ & 2,43 & $0,01^{*}$ \\
& W & Murciélago & $43,9 \%$ & 1,44 & 0,08 \\
\hline
\end{tabular}

${ }^{*} p<.05$ 
Tabla 8

Respuestas populares por evaluadora

\begin{tabular}{cclcc}
\hline Lámina & Localización & Respuesta & Asistente & Investigadora \\
\hline I & W & Murciélago & $30,6 \%$ & $50,0 \%$ \\
\hline III & D9 & Figura humana & $25,0 \%$ & $36,4 \%$ \\
\hline IV & W & Figura parahumana & $16,7 \%$ & $36,4 \%$ \\
\hline V & W & Mariposa & $47,2 \%$ & $40,9 \%$ \\
\hline V & W & Murciélago & $41,7 \%$ & $63,6 \%$ \\
\hline VIII & D1 & Figura animal entera & $55,6 \%$ & $63,6 \%$ \\
\hline X & D1 & Araña & $11,1 \%$ & $45,5 \% *$ \\
\hline
\end{tabular}

${ }^{*} p<.05$

\section{DisCusión}

Este estudio tuvo como objetivo identificar las respuestas populares para un grupo de niños asháninkas y andinos. Para ello, se contó con una muestra de 108 participantes: 58 niños asháninkas y 50 niños andinos. Sobre el tamaño óptimo de una muestra, Hernández et al. (2010) señalan que precisar este aspecto es problemático y que depende del problema de investigación y de la población por estudiar; para ello, es útil comparar el tamaño de las muestras empleadas por otros investigadores (Hernández, Fernández y Baptista, 2014). Al respecto, Meyer et al. (2007), con el objetivo de consolidar una data internacional, reúnen muestras de niños de cinco países, y ponen en evidencia la dificultad para conformar un tamaño de muestra grande. Ello, como afirma Jara (2011), puede deberse a la complejidad de la prueba, pues su correcta administración implica una capacitación larga y exigente, a lo que se suma el tiempo que demanda la aplicación misma.
En este estudio, además, buscando favorecer el rapport, la autora ha estandarizado un procedimiento que implica una entrevista y la realización de dibujos antes de administrar el Rorschach, para que en este primer momento el niño se familiarice con el evaluador y con la situación de evaluación. De esta manera, una investigación utilizando el Rorschach $\mathrm{y}$ con población infantil implica un esfuerzo mayor y un tiempo prolongado para reunir una muestra grande. De ahí que, de las muestras referidas por Meyer et al. (2007), solo la de Dinamarca fue seleccionada en forma aleatoria buscando su representatividad; el resto siguió un procedimiento similar al de este estudio: contactar primero con las instancias que permitan ubicar a los padres y a través de ellos invitar a sus hijos a participar, evaluando a quienes accedían.

De igual modo, resulta relevante considerar el impacto del evaluador en la obtención del protocolo. En este estudio, cuando la evaluadora es la investigadora, consigue un promedio de respuestas 
significativamente mayor que la asistente para la muestra de participantes: mientras la asistente obtiene tres respuestas populares, la investigadora obtiene siete (las cuatro $\mathrm{P}$ adicionales son el murciélago en la $\mathrm{W}$ de la lámina I, la figura humana en el D9 de la lámina III, la figura parahumana en la $\mathrm{W}$ de la lámina IV y la araña en el D1 de la lámina X). A la vez, la respuesta de la araña al D1 de la lámina $\mathrm{X}$ es significativamente mayor cuando la evaluadora es la investigadora. Al respecto, Exner (1994) reporta que los evaluadores con mayor experiencia obtienen más protocolos de longitud media. A su vez, Lis, Parolin, Calvo, Zennaro y Meyer (2007) encuentran que los sujetos examinados por evaluadores expertos lucen más complejos, articulados y comprometidos con la tarea que los examinados por evaluadores con un menor entrenamiento y experiencia con la encuesta, lo que puede tener un gran efecto en los puntajes del SCR. De este modo, a diferencia de lo planteado por Baughman (como se citó en Exner, 1994), en este estudio el evaluador sí tiene un efecto en las respuestas populares, pese a que estas son las más fáciles de reconocer al basarse en las características más evidentes de las manchas (Exner, 2000).

Otro factor del impacto del evaluador es la motivación; si el evaluador realiza la evaluación buscando conocer de manera más precisa o profunda al evaluado, es previsible que favorezca que este último se tome el tiempo necesario para elaborar sus respuestas y que luego, en la encuesta, el evaluador ensaye diferentes estrategias para aclarar las respuestas del evaluado. En contraste, un evaluador que ve la evaluación como una tarea puede estar más orientado a resolverla con prontitud, lo que puede generar protocolos con menos respuestas y una menor elaboración. En este estudio, la moda de las respuestas obtenidas por la asistente es de 14, lo que resulta el número mínimo para que el protocolo pueda ser considerado válido. Por su parte, la baja presencia de la respuesta de la araña en el D1 de la lámina X en los protocolos tomados por la asistente puede responder a una menor exploración de esta lámina, que es la última, por parte de los evaluados. Al respecto, es probable que al alcanzar el número mínimo de respuestas para que el protocolo resulte válido se genere algún tipo de comunicación no verbal que transmita el mensaje de haber concluido con la tarea. Considerando la habilidad y experiencia de la asistente en el manejo del Rorschach, este mensaje parece darse sin que se percate de ello.

Lo anterior pone de relieve no solo la importancia de la experiencia del evaluador, sino también su aproximación a la situación de evaluación. Por eso, ambos aspectos deben ser considerados al momento de capacitar a examinadores con el Rorschach, particularmente cuando los evaluados son niños y cuando estos se diferencian culturalmente del evaluador. En efecto, es probable que los niños, al estar menos habituados a los contextos de evaluación, requieran de un mayor tiempo para elaborar y resolver la situación inestructurada y ambigua que implica la prueba, incluso ante los rasgos estimulares más distintivos de las manchas que dan lugar a la respuesta popular. A la vez, los 
evaluados que se diferencian culturalmente del evaluador pueden requerir de una mayor disposición y tiempo por parte de este último para sentirse cómodos en la situación de evaluación.

Tomando en cuenta que en este estudio el impacto del evaluador se refleja tanto en la cantidad como en el tipo de la respuesta brindada por los niños de un contexto cultural específico, pasamos a discutir los hallazgos en torno a la respuesta popular. Así, observamos que de las 13 respuestas populares listadas en el sistema comprehensivo del Rorschach, solo tres respuestas singulares alcanzan una frecuencia de al menos $33 \%$, y de ellas, solo dos tienen una proporción significativa más allá de la muestra de participantes. Al respecto, es importante destacar que para hallar estas respuestas se han revisado todos los contenidos de las 2465 respuestas brindadas por los participantes; por eso, independientemente de las populares listadas por el SCR, se puede afirmar que las populares reportadas en esta investigación son, en efecto, los contenidos que aparecen en al menos uno de cada tres protocolos.

En ese sentido, consideramos necesario mantener el tratamiento individualizado de las respuestas populares, evitando agruparlas cuando se trata de contenidos conceptualmente diferentes (por ejemplo, la figura humana y la figura parahumana en las láminas III, IV y IX) o cuando la respuesta se ubica en diferentes áreas de la mancha (por ejemplo, la figura parahumana en la $\mathrm{W}$ y el D7 de la lámina IV). Ello, porque de manera individualizada estas respuestas obtienen porcentajes que difieren entre sí y que no alcanzan el porcentaje para ser consideradas como una popular (por ejemplo, en la lámina IV, la figura parahumana tiene en la $\mathrm{W}$ una frecuencia de $24,1 \%$, mientras que en el D7 su presencia es de 9,3\%); y porque tampoco cumplirían con el criterio estadístico de diferenciarse de otras respuestas con una frecuencia alta (Exner, 1994). En este aspecto, Jacquemin (como se citó en Lelé, 2007) afirma que cada respuesta popular tiene un peso con relación a su frecuencia y a su grado de especificidad.

De otro lado, Lelé (2007) señala que la lista de respuestas populares puede variar de una cultura a otra, de acuerdo con factores como el contexto y la mentalidad de la población; y esta respuesta es la que mejor expresa las diferencias culturales. En este sentido, comparando diferentes estudios, Lunazzi (2006) señala que solo las muestras de Exner (Estados Unidos) y Sendín (Madrid) reportan el listado de 13 respuestas populares, en tanto que las muestras de Campo (Barcelona) y Mattlar (Finlandia) cuentan con siete populares. Por su parte, Lelé en un estudio con 100 sujetos no pacientes entre los 18 y 36 años en el estado de Minas Gerais (Brasil) halla seis respuestas que cumplen con el criterio para ser consideradas populares. Por su parte, en muestras de niños, Jara (2005) obtiene cuatro respuestas populares en menores andinos de Apurímac (Perú) y cinco en una muestra limeña (Jara, 2015); a su vez, Loureiro (2010) en una muestra de 180 niños de 9 a 11 años de São Paulo (Brasil) encuentra solo dos respuestas con una presencia de 
al menos el $33 \%$ (el animal cuadrúpedo en el D1 de la lámina VIII y las figuras humanas de la lámina III).

Como se aprecia, si bien inicialmente el número de respuestas populares ubicadas en este estudio puede ser considerado bajo con relación al listado del SCR, cuando se revisan otras muestras esta diferencia se hace menos evidente. Se confirman, además, la inestabilidad de algunas puntuaciones en la evaluación de los menores y la necesidad de realizar estudios normativos (Meyer et al., 2007), en este caso, de la lista de respuestas populares, pese a que la $\mathrm{P}$ es considerada uno de los signos más estables del SCR (Exner, 1994). Esto se debe no solo a que puede ser poco válido evaluar a los niños empleando indicadores desarrollados bajo otros criterios de crianza y otras condiciones del entorno, sino también por lo que se logra conocer respecto al proceso del menor de ir construyendo ese modo común de percibir las cosas (León, 1983).

De esta manera, analizando las respuestas populares halladas en los participantes, observamos que estas corresponden a tres de las cuatro que Exner (2000) reporta como las de mayor frecuencia en la lista de P del SCR: el animal en el D1 de la lámina VIII; y la mariposa y el murciélago en la $\mathrm{W}$ de la lámina $\mathrm{V}$ (la segunda de mayor frecuencia para Exner es la figura humana en el D9 de la lámina III). A su vez, Lunazzi (2006) también observa que las únicas respuestas populares presentes en todas las muestras que revisa - por lo que considera que estas tienen un carácter "universal"- - son la figura humana o su representación en el
D9 de la lámina III, el murciélago en la W de la lámina $\mathrm{V}$ y el animal cuadrúpedo en el D1 de la lámina VIII. Esto va en la línea de lo señalado por Exner (1994) sobre la improbabilidad de que las respuestas con mayor frecuencia sufran variaciones transculturales. En tal sentido, en estas respuestas lo que parece primar en la tarea de desidentificar el estímulo de la mancha es el indicio distal crítico de la forma (la lámina $\mathrm{V}$ es la más estructurada de todas y el D1 de la lámina VIII también tiene una forma bastante definida) y la familiaridad e interés de los menores por los contenidos animales (en este estudio, los contenidos de la figura animal completa son los más reportados: $47,44 \%$ ).

La escasa presencia de respuestas populares en los menores de estas comunidades rurales, una de la selva alta y otra del Ande, puede responder a la etapa evolutiva, al entorno cultural y al sexo, además del impacto del evaluador ya señalado. Respecto a la variable evolutiva, observamos que si bien para el grupo de participantes existen tres respuestas populares entre los 6 y 11 años, cuando se observa si la proporción obtenida es significativa más allá de la muestra, se encuentra que entre los 6 y 7 años no existe ninguna respuesta popular, mientras que los de 8 y 9 años tienen una respuesta $\mathrm{P}$ (el animal en el D1 de la lámina VIII), y los de 10 y 11 años, dos respuestas $P$ (el animal en el D1 de la lámina VIII y la mariposa en la $\mathrm{W}$ de la lámina $\mathrm{V}$ ). Se confirma, por tanto, lo indicado por Ames et al. (1971, como se citó en Exner, 1994) con respecto a que las $\mathrm{P}$ se incrementan gradualmente a medida que el niño crece. 
A la vez, de manera descriptiva, se aprecia que la frecuencia de las $\mathrm{P}$ también se incrementa paulatinamente. Así, la respuesta animal en el D1 de la lámina VIII pasa de $48 \%$ entre los 6 y 7 años a 57,1 \% entre los 8 y 9 años, y se mantiene en un porcentaje similar entre los 10 y 11 años (56,1\%). La mayor facilidad para decodificar tempranamente esta respuesta puede deberse a otro de los indicios distales: el color rojo. Al respecto, Jiménez, De Diego y Sánchez (1994) aluden a la atracción universal que los niños experimentan por el color rojo. Por su parte, Exner (1994) afirma que el color tiene un gran impacto en la formulación de respuestas $\mathrm{y}$, citando diferentes investigaciones (Schachtel, 1943; Rickers-Ovsiankina, 1943; Shapiro, 1956, 1960), plantea que la percepción del color implica un proceso con una menor actividad cognitiva; por tanto, es un proceso más pasivo e inmediato que la forma. Sin embargo, esto no implica que el color sea articulado en la verbalización (Exner, 2000) o decodificado como determinante, pero sí que, en esta lámina cromática, la percepción del color rojo parece llamar la atención del evaluado sobre el resto del campo estimular. En este sentido, León (1983) plantea que, para ser elicitado, el estímulo específico tiene que localizarse de manera saliente en la mancha. Es decir, para los menores participantes, el D1 de la lámina VIII puede haberse destacado por su color rojo; luego, permite la formulación de una respuesta común por su estructuración formal que asemeja a un animal cuadrúpedo. Es probable que la frecuencia de esta respuesta siga incrementándose conforme la persona crece, ya que Exner (2000) reporta que más del $90 \%$ de los individuos brinda esta respuesta.

En tanto, la respuesta de mariposa en la $\mathrm{W}$ de la lámina $\mathrm{V}$, presente en alrededor del $40 \%$ de los niños entre los 6 y 9 años, pasa a 51,2 \% entre los 10 y 11 años (su presencia en la muestra total es de 44,4\%). Por lo que a esto se refiere, Jara (2015) encuentra en una muestra de 346 niños limeños entre los 6 y 11 años que esta respuesta está presente en el 51,5\% de los menores, mientras que Exner (2000) reporta que el $44 \%$ de las personas genera esta respuesta. Como se aprecia, estos resultados parecen indicar que por lo menos desde los 10 años la respuesta de mariposa en la $\mathrm{W}$ de la lámina $\mathrm{V}$ tiene una frecuencia que se ubica dentro de un rango relativamente estable a lo largo de los años.

Sin embargo, considerando el sexo, se observa que la respuesta de mariposa en la $\mathrm{W}$ de la lámina $\mathrm{V}$ alcanza el criterio para ser considerada popular de manera significativa (es decir, más allá de la muestra) solo en el caso de los varones $(51,8 \%)$, aunque para la muestra de niñas participantes sí lo sería (36,5\%). En contraste, Jara (2015) reporta en una muestra de menores limeños que el porcentaje de mujeres que brinda esta respuesta es de 55,9\% versus el 45,7 \% de los hombres. Al respecto, pese a que las diferencias halladas entre las respuestas de los niños y las niñas no llegan a ser significativas (además de esta respuesta, 
en la respuesta animal del D1 de la lámina VIII, los varones obtienen $60,7 \%$ versus el 48,1 \% de las niñas), a nivel descriptivo estos datos podrían ser el indicio de una mayor dificultad en el caso de las niñas rurales, con respecto a los varones de la muestra y a sus pares limeñas, para alcanzar un procesamiento más ajustado de la información provista por el entorno, reconociendo los datos más evidentes para luego hacerlos coincidir con los elementos disponibles en su memoria (Exner, 2000). Esto hace necesario el estudio de la calidad formal a fin de valorar si las respuestas brindadas por los menores se ajustan perceptivamente a la mancha. En tanto, dado que todos los participantes de este estudio se encuentran escolarizados, esta mayor dificultad podría estar asociada a que las niñas son menos estimuladas a explorar e incorporar activamente los diferentes estímulos que ofrece su contexto. En esa línea, Mogrovejo (2001) halla que en el colegio, uno de los espacios más importantes de socialización para los niños, se concede mayor libertad para desplazarse, moverse y proveerse de recursos a los varones; por el contrario, las niñas son rápidamente reprendidas cuando se mueven o comportan indebidamente, por lo que no sorprende que ellas se inclinen a convertirse en observadoras pasivas de lo que sucede a su alrededor.

De otro lado, con respecto a la respuesta de murciélago en la $\mathrm{W}$ de la lámina $\mathrm{V}$, si bien esta resulta popular para los participantes (37\%), es más preciso indicar que lo sería a partir de los 8 años (38,1 \% entre los 8 y 9 años, y 43,9 \% entre $\operatorname{los} 10$ y 11 años), no antes. Sin embargo, esta respuesta resulta popular para los niños asháninkas (50\%), incluso más allá de la muestra; pero no para los niños andinos del Cusco (22\%), lo que marca una diferencia significativa entre ambas comunidades. Además, para los niños asháninkas, la respuesta de murciélago tiene una presencia mayor que la respuesta de mariposa (44,8\%); a lo que se agrega que el mismo contenido en la lámina I cumple con el criterio para ser considerado popular (37,9\%) entre estos menores. Al respecto, Jara (2006), en un estudio con niños andinos de Apurímac, no encuentra que el murciélago sea una respuesta popular ni en la lámina I ni en la V. La misma autora (Jara, 2015) reporta en su estudio con niños limeños que, solo para los de nivel socioeconómico bajo, el murciélago de la lámina I resulta popular. Este hecho parece indicar el impacto del contexto cultural en el que se ubican los menores; así, es probable que los niños asháninkas estén más familiarizados con este animal que los niños andinos del Cusco. En la misma línea, León (1983) señala que los miembros de las muestras deben haber estado expuestos al conocimiento de los objetos mencionados.

Con relación a las comunidades, encontramos que entre los niños asháninkas cuatro respuestas alcanzan el criterio para ser consideradas populares, de las cuales tres tienen una proporción suficiente para serlo más allá de la muestra. En cambio, entre los niños andinos del Cusco, solo dos respuestas logran el criterio de un tercio y de ellas 
solo una tiene una proporción suficiente para considerarla popular más allá de la muestra. Asimismo, si consideramos el impacto de la evaluadora, observamos que, cuando esta es la investigadora, los niños asháninkas obtienen tres respuestas populares más: la figura humana en el D9 de la lámina III, la figura parahumana en la W de la lámina IV y la araña en el D1 de la lámina $\mathrm{X}$. Dado que todos los niños andinos del Cusco fueron evaluados por la investigadora, si tomamos en cuenta solo a los niños evaluados por ella, las diferencias entre las comunidades se acrecienta. Esta diferencia se refiere a las respuestas populares, pero no al número promedio de respuestas ni a la frecuencia de los contenidos brindados por los participantes de ambas comunidades. En tal sentido, estos resultados parecen indicar una menor disposición de los niños andinos del Cusco, versus sus pares asháninkas, para percibir, reconocer $\mathrm{y}$ generar los mismos significados que su comunidad sociocultural; es decir, para ver el mundo como los demás (Lunazzi, 2006). Esto puede responder a una mayor inhibición o a una posición más pasiva frente al entorno.

Al respecto, Villapolo y Vásquez (1999) señalan que los asháninkas promueven la autonomía en sus niños, lo que puede traducirse en una mayor libertad para jugar y explorar su entorno; por su parte, la cultura andina, al valorar lo útil y lo pragmático, pone de relieve la fuerza de trabajo de los menores, asignándoles tareas que les permitan contribuir con sus familias (Anderson, 1994; Ortiz, 1993, 1994). A la vez, Anderson (1994) plantea que los padres andinos tienden a ser exigentes, enfatizando en la obediencia y la responsabilidad. Todo ello puede llevar a que estos niños tengan menos espacio para el juego, y a que las normas y convenciones les sean transmitidas de un modo vertical, lo que los limita que vayan descubriendo, ensayando, probando e interiorizando de un modo más vivencial y desde ellos mismos la forma como los demás comprenden y asimilan lo que sucede en su contexto.

En cuanto a las tres respuestas adicionales que resultan populares entre los participantes asháninkas cuando la evaluadora es la investigadora, ya se analizó el caso de la respuesta de la araña en el D1 de la lámina X; por tanto, pasaremos a revisar la respuesta de la figura humana en el D9 de la lámina III y la respuesta de la figura parahumana en la $\mathrm{W}$ de la lámina IV. En el caso de la respuesta de la figura humana en el D9 de la lámina III, esta es la segunda más frecuente (más del $80 \%$ ) reportada por Exner (2000). Por su parte, Jara (2006) plantea que esta es una de las cuatro respuestas populares halladas en una muestra de niños andinos de Apurímac, que abarca menores de zonas urbanas y rurales. La misma autora (Jara, 2015) señala, en una muestra de niños limeños, que esta respuesta se incrementa gradual y significativamente conforme el niño crece, siendo $\mathrm{P}$ a partir de $\operatorname{los} 8$ años; a su vez, considerando el grupo total, solo sería P para las niñas $(49,8 \%$ versus 29,2 \%). En tal sentido, es probable que la escasa presencia de la respuesta de la figura humana de la lámina III entre los participantes de este estudio tenga 
que ver con una mayor dificultad para alcanzar el desarrollo esperado en su aproximación a los otros; especialmente, en el caso de las niñas y en el caso de los niños andinos y rurales del Cusco. Dado el impacto del evaluador, esta respuesta también podría ser sensible al rapport establecido con los menores.

Respecto a la respuesta de la figura parahumana en la $\mathrm{W}$ de la lámina IV, tanto en los participantes asháninkas como en los andinos del Cusco, encontramos que ella resulta popular para los más pequeños de la muestra (6 y 7 años). Al respecto, los menores brindan algunos contenidos culturales específicos; así, los niños asháninkas mencionan al chullachaqui, al pishtaco, al machetero, al tuco (diablo) y al volador ("come gente"); mientras los niños cusqueños se refieren al paracucucho ("que se come el alma"), el kukuchi (condenado) y el mal espíritu. Esto puede ser un indicador de una aproximación temerosa y fantasiosa entre los niños más pequeños hacia los otros, la misma que no evoluciona hacia una presencia más real y objetiva. Ello queda reforzado por la baja presencia de la respuesta de la figura humana en el D9 de la lámina III.

De este modo, los resultados del presente estudio nos llevan a plantear que, en el proceso de mirar el mundo como lo hacen los demás, los niños participantes requieren que el estímulo llame su atención y sea fácil de identificar (por su estructuración formal y por la familiaridad que se tiene con él), y que, además, se sientan cómodos y acompañados mientras realizan esta tarea de reconocimiento. Ese modo de pensar común es progresivo y poco extenso durante la infancia media. También es probable que se limite ante un entorno carenciado y donde los patrones de crianza y de relación restrinjan el juego que hace posible el despliegue $\mathrm{y}$ la exploración activa de los niños de su contexto y de la información que tienen a su alcance, lo que parece afectar sobre todo a las niñas y a los menores andinos de zonas rurales.

Como se aprecia, las respuestas populares permiten conocer no solo cómo los niños van procesando e incorporando la información de su medio, sino también el modo diferenciado en que lo hacen según su género, contexto cultural, momento evolutivo y los otros que lo acompañan en ese proceso. Con relación a esos otros, se refuerza la importancia de la experticia del evaluador en el manejo de la prueba, pero también su actitud y motivación al momento de la evaluación. A partir de este estudio, podemos señalar la necesidad de revisar los signos del SCR, aun los más estables, no solo en cuanto a la codificación, sino también a la interpretación, a fin de evitar el riesgo de comparar equivocadamente a nuestros menores con niños que se han desarrollado en contextos culturales distintos.

Los resultados de esta investigación proveen del listado de las respuestas populares esperadas en niños peruanos de dos contextos culturales específicos, tomando en cuenta, además, el sexo y la edad. Estos resultados permiten avanzar en la adaptación del Rorschach en nuestro medio, brindando información precisa y objetiva sobre el ajuste y la 
adaptación de los niños al modo común de ver las cosas en su cultura. Esto puede servir para diseñar programas orientados al mejoramiento de la calidad de vida de nuestros niños, favoreciendo la exploración lúdica y activa de su medio, con la expectativa de contribuir a la formación de una nueva generación que se ajuste más efectivamente a su contexto, a pesar de las carencias que pueda plantear la realidad.

\section{Referencias}

Alcalde, A. (2001). Estudio del control $y$ tolerancia del estrés en los niños de la calle de Lima Metropolitana a través del sistema comprehensivo de Exner-Rorschach (tesis de maestría). Universidad Nacional Mayor de San Marcos, Lima.

Alessandro, M., Alonso, H., y Passalacqua, A. (septiembre, 2000). Sistema comprensivo y escuela argentina de Rorschach. Similitudes y diferencias. Comunicación libre en el VI Congreso Europeo de Rorschach (ERA). París. Recuperado de http://www. asoc-arg-rorschach.com.ar/docs/Trabajo_Exner.pdf

Allen, J., y Dana, R. (2004). Methodological Issues in Cross-Cultural and Multicultural Rorschach Research. Journal of Personality Assessment, 82(2), 189-206.

Ampuero, A. (2000). Autoestima en niños diabéticos de ambos sexos a través del psicodiagnóstico de Rorschach (tesis de licenciatura). Pontificia Universidad Católica del Perú, Lima.

Anderson, J. (1994). La socialización infantil en comunidades andinas $y$ de migrantes urbanos en el Perú. Proyecto de innovaciones pedagógicas no formales. Lima: Ministerio de Educación; Fundación Bernard van Leer.

Bower, T. (1984). El mundo perceptivo del niño (3. ${ }^{\mathrm{a}}$ ed.). Madrid: Ediciones Morata.

Córdoba, A. (2006). Desarrollo cognitivo. En A. Córdoba, A. Descals, y M. Gil (Eds.), Psicología del desarrollo en la edad escolar (pp. 89-116). Madrid: Ediciones Pirámide.

Cornejo, M. (2005). Características de personalidad en niños talentosos limeños que viven en condiciones de pobreza, a través del psicodiagnóstico de Rorschach (tesis de licenciatura). Pontificia Universidad Católica del Perú, Lima.

Dana, R. (2009). Multicultural Assessment. Principles, Aplications and examples. Nueva York: Psychology Press.

Daroglou, S. (2009). Greek Assessees (Rorschach CS). En R. Dana (Ed.), Multicultural Assessment. Principles, Applications, and Examples. Nueva York: Psychology Press.

Del Castillo, M. (2014). Relaciones interpersonales en niños de familias desplazadas por el conflicto armado interno (Tesis de licenciatura). Pontificia Universidad Católica del Perú, Lima. 
Delclaux, I. (1982). Psicología cognitiva y procesamiento de la información. Madrid: Ediciones Pirámide.

Eldad, L. (2009). Controles y tolerancia al estrés en un grupo de niños diagnosticados con cáncer (tesis de licenciatura). Pontificia Universidad Católica del Perú, Lima.

Ephraim, D. (1996). El método Rorschach en la actualidad. Caracas: Fondo Editorial de la Facultad de Humanidades y Educación de la Universidad Central de Venezuela; Monte Ávila Editores Latinoamericana.

Exner, J. E. (1994). El Rorschach. Un sistema comprehensivo. Volumen 1: Fundamentos básicos. Madrid: Psimática.

Exner, J. E. (1996). Critical Bits and the Rorschach Response Process. Journal of Personality Assessment, 67(3), 464-477.

Exner, J. E. (2001). Manual de codificación del Rorschach para el sistema comprehensivo (5. ${ }^{\mathrm{a}}$ ed.). Madrid: Psimática.

Exner, J. E. (2000). Principios de interpretación del Rorschach. Un manual para el sistema comprehensivo. Madrid: Psimática.

Exner, J. E., y Sendín, C. (1998). Manual de interpretación del Rorschach para el sistema comprehensivo (2. ${ }^{a}$ ed.). Madrid: Psimática.

Exner, J. E., y Weiner, I. (1995). The Rorschach: A Comprehensive System. Volume 3: Assessment of children and adolescents. Nueva York: John Wiley and Sons.
Franchi, V., y Andronikof-Sanglade, A. (1999). Methodological and Epistemological Issues Raised by the Use of the Rorschach Comprehensive System in Cross-Cultural Research. En A. Andronikof-Sanglade (Ed.), Rorschachiana XXIII. Yearbook of the International Rorschach Society (pp. 118-134). Gotinga: Hogrefe and Huber Publishers.

Hernández, R., Fernández, O., y Baptista, O. (2010). Metodología de la

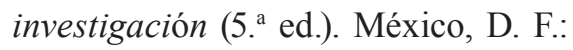
McGraw-Hill; Interamericana.

Hernández, R., Fernández, O., y Baptista, O. (2014). Metodología de la investigación (6. ${ }^{\mathrm{a}}$ ed.). México, D. F.: McGraw-Hill; Interamericana.

Jara, L. (2000). Conociendo a los niños andinos a través de sus dibujos y sueños. En R. Panez, G. Silva, y M. Silva (Eds.), Resiliencia en el Ande (pp. 249284). Lima: Panez y Silva Ediciones.

Jara, L. (agosto, 2006). Respuestas populares en niños andinos. Un estudio sobre el ajuste perceptivo y normativo. Trabajo presentado en el XIII Congreso Latinoamericano de Rorschach y Métodos Proyectivos, Lima.

Jara, L. (2008). Representaciones sobre el maltrato infantil en niños limeños $y$ andinos a través de sus dibujos (Tesis de maestría). Pontificia Universidad Católica del Perú, Lima.

Jara, L. (2011). La administración del Rorschach: la complejidad e integralidad del método. Persona, 14, 111-126. 
Jara, L. (julio, 2015). Ajuste perceptivo y normativo en niños a través de las respuestas populares en el Rorschach. Trabajo presentado en el $X X X V$ Congreso Interamericano de Psicología, Lima.

Jiménez, F., De Diego, R., Sánchez, G. (1994). Color rojo y test de Rorschach. Psiquis, 15(10), 35-45. Recuperado de http://campus.usal.es/ petra/Profe sores/GuadalupeSanchez/Revistas /Color\%20rojo\%20y\%20Rorschach \%20(1994).pdf

Lafuente, M., y Gil, M. (2006). Desarrollo sensorioperceptual. En A. Córdoba, A. Descals, y M. Gil (Eds.), Psicología del desarrollo en la edad escolar (pp. 55-71). Madrid: Ediciones Pirámide.

Lelé, Á. (2007). Perception of Reality and Norm: A Study of the Popular Responses to Rorschach's psychodiagnostis in Brazil. Rorschachiana, 28, 81-99.

León, F. (1983). El concepto de respuesta popular Rorschach y su validez. Revista de Psicología de la Pontificia Universidad Católica del Perú, 1(2), 73-86.

Lis, A., Parolin, L., Calvo. V., Zennaro, A., y Meyer, G. (2007). The Impact of Administration and Inquiry on Rorschach Comprehensive System Protocols in a National Reference Sample. Journal of Personality Assesment, 89(S1), S193-S200.

Loureiro, R. (2010). Psicodignóstico de Rorschach en niños de 9 a 11 años: un estudio normativo (tesis de maestría). São Paulo: Universidad de São Paulo.

Lunazzi, H. (2006). Comparaciones interculturales respecto del conformismo cognitivo y social. Psicodiagnosticar. Recuperado de http://www.psico. unlp.edu.ar/catedras/psicodiagnostico/lectura.html

Meyer, G., Erdberg, P., y Shaffer, T. (2007). Toward International Normative Reference Data for the Comprehensive System. Journal of Personality Assessment, 89, 201-216. doi: 10.1080/00223890701629342

Mogrovejo, S. (2001). Promovamos la equidad de género en el aula. Lima: Ministerio de Educación.

Nakamura, N., Fuchigami, Y., y Tsugawa, R. (2007). Rorschach Comprehensive System Data for a Sample of 240 Adult Non-Patients from Japan. Journal of Personality Assessment, 89(S1), S97-S102.

Ortiz, A. (1993). La pareja y el mito. Estudio sobre las concepciones de la persona y de la pareja en los Andes. Lima: Fondo Editorial de la Pontificia Universidad Católica del Perú.

Ortiz, A. (1994). Un estudio sobre los grupos autónomos de niños a partir de un trabajo de campo en Champaccocha, Andahuaylas. Proyecto de innovaciones pedagógicas no formales. Lima: Ministerio de Educación; Fundación Bernard van Leer.

Puga, L. (2008). Relaciones interpersonales en un grupo de niños que 
reciben castigo físico y emocional, medidas a través del psicodiagnóstico de Rorschach (tesis de licenciatura). Pontificia Universidad Católica del Perú, Lima.

Ráez, M. (agosto, 2006). Situación de las pruebas de personalidad en el Perú. Estudio comparativo 1997-2006. Comunicación presentada en $\mathrm{La}$ situación de las pruebas psicológicas en Latinoamérica. Simposio llevado a cabo en el XIII Congreso Latino Americano de Rorschach y Métodos Proyectivos. Lima.

Rivarola, M. (2015). Afectividad y control y tolerancia al estrés en niños(as) víctimas de maltrato (tesis de licenciatura). Pontificia Universidad Católica del Perú, Lima.

Rivera, S. (2000). Relaciones objetales en niños maltratados y abusados utilizando el psicodiagnóstico de Rorschach (Exner) y la escala de mutualidad de autonomía (Urist) (tesis de licenciatura). Pontificia Universidad Católica del Perú, Lima.

Sendín, C. (2007). Manual de interpretación del Rorschach para el sistema comprehensivo (3. ${ }^{\mathrm{a}}$ ed.). Madrid: Psimática.

Shaffer, T., Erdberg, P., y Meyer, G. (2007). Introduction to the JPA Special Supplement on International Reference Samples for the Rorschach Comprehensive System. En T. Shaffer,
P. Erdberg, y G. Meyer (Eds.), Journal of Personality Assessment [Special Issue: International Reference Samples for the Rorschach Comprehensive System], 89(S1), S2-S6.

Smith, B. (1994). Object Relations Theory and the Integration of Empirical and Psychoanalytic Approaches to Rorschach Interpretation. En I. Weiner (Ed.), Rorschachiana XIX (pp. 61-77). Berna: Hogrefe and Huber Publishers.

Timyan, J. (1988). Cultural Aspects of Psychosocial Development: An Examination of West African Childrearing Practices [Informe preparado para el Taller Regional de UNICEF]. Recuperado de http://www.ecdgroup.com/ download/aalcapda.pdf

Vargas, V. (2003). Características psicológicas de niños y niñas que trabajan en dos centros mineros artesanales de Perú. Recuperado de https:// es.scribd.com/document/78109280/ Caracteristicas-Psicologicas-de-Ninios-y-Ninias-Que-Trabajan-en-Mineria-Artesanal-Version-Final

Viglione, D., y Hilsenroth, M. (2001). The Rorschach: Facts, Fictions, and Future. Psychological Assessment, 13(4), 452-471.

Villapolo, L., y Vásquez, N. (1999). Entre el juego y la guerra: recursos psicológicos y socioculturales de los niños asháninkas ante la violencia política. Lima: CAAAP. 
